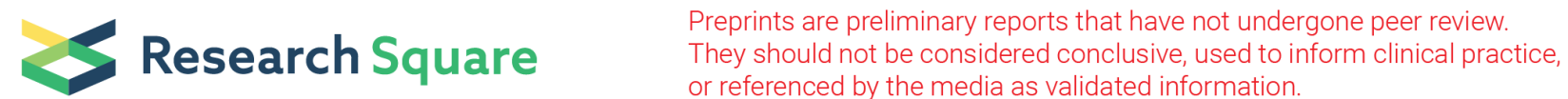

\section{The impact of information sources on COVID-19 Vaccine hesitancy and resistance in Sub- Saharan Africa}

\author{
Uchechukwu L Osuagwu ( $\sim$ l.osuagwu@westernsydney.edu.au ) \\ Western Sydney University \\ Khathutshelo P Mashige \\ Westville Campus, African Vision Research Institute, University of KwaZulu-Natal

\section{Godwin Ovenseri-Ogbomo}

University of the Highlands and Islands

Esther Awazzi Envuladu

University of Jos

\section{Emmanuel Kwasi Abu}

University of Cape Coast

\section{Chundung Asabe Miner}

University of Jos

Chikasirimobi G Timothy

Masinde Muliro University of Science and Technology

\section{Bernadine N Ekpenyong}

University of Calabar, Cross River State

\section{Raymond Langsi}

University of Bamenda

Onyekachukwu M Amiebenomo

Cardiff University

\section{Richard Oloruntoba}

Curtin University

Piwuna Christopher Goson

University of Jos

\section{Deborah Donald Charwe}

Tanzania Food and Nutrition Center

\section{Tanko Ishaya}

University of Jos

\section{Kingsley E Agho}

Western Sydney University 
Keywords: Coronavirus, Facebook, Media, Africa, Television, Misinformation, Survey, Radio, Healthcare workers, Lockdown

Posted Date: February 24th, 2022

DOI: https://doi.org/10.21203/rs.3.rs-1385351/v1

License: @ (i) This work is licensed under a Creative Commons Attribution 4.0 International License. Read Full License 


\section{Abstract \\ Background}

Vaccination remains the most powerful weapon against the emergence of new variants of coronavirus (COVID-19). However, false information about COVID-19 vaccines through various platforms including social media remain a major threat to global public health. The study examined the impact of information sources on COVID-19 vaccine hesitancy and resistance in sub-Saharan Africa (SSA).

\section{Methods}

A validated web-based questionnaire was administered in French and English to 2572 participants from subSaharan African (SSA) countries including Cameroon, Ghana, Kenya, Mozambique, Nigeria, South Africa, Tanzania, who were aged 18 years and over. An e-link to the survey was disseminated via emails and posted on social media platforms (Facebook and WhatsApp). The COVID-19 vaccination status (vaccinated, hesitancy and resistance) and their association with sources of information (mainstream media and social media) were assessed by conducting multinomial logistic regression analyses.

\section{Results}

The prevalence of vaccine resistance and hesitancy in SSA was higher among social media users (72.2 and $84.5 \%$ ) and those that watched TV (73.0 and 79.7\%), but lower among newspaper readers ( 42.5 and $36.7 \%$ ) respectively, during the pandemic. Multivariate analysis revealed that Southern and Central African respondents were more likely rely on TV for information, women and those who smoke were more likely to rely on friends and family members for information during the pandemic. Lower education (primary education or less) was associated with lower likelihood of social media use for COVID-19 related information compared with postgraduate qualification (aOR $0.36,95 \% \mathrm{Cl} 0.20,0.62$ ). Resistance to COVID-19 vaccination was associated with reliance families and friends $(\mathrm{aOR} 1.44,95 \% \mathrm{Cl}=1.08,1.93)$, watching $\mathrm{TV}(\mathrm{aOR} 1.54,95 \% \mathrm{Cl}=$ $1.11,2.14$ ) and social media use (adjusted odds ratio aOR $2.93,95 \% \mathrm{Cl}=2.10,4.09$ ), which was also associated with vaccine hesitancy $(\mathrm{aOR} 1.46,95 \% \mathrm{Cl}=1.15,1.85)$ among the respondents.

\section{Conclusions}

Our study showed that reliance on social media and television had the strongest impact on vaccine hesitancy. However, further research and investments are needed to improve the availability of reliable healthcare information in these channels to protect people and empower them to make appropriate choices about their health.

\section{Introduction}

The COVID-19 pandemic has significantly impacted economic, health and living conditions on the African continent and elsewhere [1, 2]. The impact on individuals, families and communities across Africa has been 
unprecedented. While the global economic loss is still unfolding, it is projected to be quite huge particularly in African countries [3]. The risk of COVID-19 resurgence remains high in several African countries due to poor adherence to public health measures, mass gatherings, low testing and vaccination rates [4]. Vaccination remains the most powerful weapon against the emergence of new variants[5] as well as reaching herd immunity[6], but compared with the rich European and North-American countries, African countries have barely initiated the vaccination program[7] with only $11 \%$ of the adult population fully vaccinated[5].

The African continent has witnessed four waves of COVID-19 over the last two years and has improved in their capacity to manage COVID-19 cases [5]. The region has also witnessed significant increase in the supply of COVID-19 vaccines and has received around 672 million doses of COVID-19 vaccines mostly facilitated by COVAX (65\%) and the rest through bilateral deals (29\%) and the African Union's Vaccines Acquisition.

Exposure time to COVID-19-related news increased over time during the pandemic [8] and more exposures to news have direct implications on people's action such that receiving timely and informative communication during a time of uncertainties promotes public cooperation [9].

Vaccine hesitancy (reluctance to receive vaccines) is one of the top ten threats to global health [10] and this is fuelled by health information obtained from the news media, internet and social media platforms [11-14]. Vaccine hesitancy is also high among certain population groups $[15,16]$ probably due to the previous medical experiment amongst these population group[17] and poor messaging [18]. Misinformation regarding the benefits, medicinal composition, and adverse effects of vaccination, limits patient understanding and overall buy-in [11]. Although access to technology has improved during the pandemic, and the use of social media has increased [11], there are concerns for the spread of misinformation across different social networks propagated via the contemporary anti-vaccination movement, to fuel vaccine hesitancy $[19,20]$. This has the potential to compromise public confidence in the COVID-19 vaccine for the prevention of the disease [21]. However, where social media platforms were used to propagate healthy messages, by nurses and doctors, authors observed a significant improvement in compliance to public health messages and subsequent COVID19 infections [14].

Sources of vaccination information have different effects on people's coping appraisal of COVID-19 vaccination [13]. Unlike mainstream media, social media such as Facebook, Twitter, Instagram, WhatsApp, and Pinterest allow individuals to rapidly create and share content globally without editorial oversight [22, 23]. These are complex and fluid ecosystems, on which anti-vaccination viewpoints can be amplified and represented as mainstream, and vaccine-hesitant parents can encounter compelling narratives from other parents dissuading vaccination [24]. Misinformation and unsubstantiated rumours regarding COVID-19 and potential vaccination against SARS-CoV-2 have already begun emerging on social media platforms, threatening to erode public confidence as the vaccines are rolled out in African countries [25]. Information spread through social media directly or indirectly increases hesitancy towards COVID-19 vaccination, while the opposite effect was observed for institutional websites [20]. Since social media platforms may self-select content streams, contributing to ideological isolation, it is important that owners ensure that social media platforms provide access to accurate information on the safety and efficacy of vaccinations [22]. 
The uptake of COVID-19 vaccination in Sub-Saharan Africa (SSA) may be impeded by the rapid spread of misinformation on social media leading to belief in false rumors about the pandemic [22], which has been associated with poor health seeking behavior $[26,27]$. The recent mixed international messages about the efficacy of the different COVID-19 vaccines, their side-effects beyond the local and systemic effects [28, 29] and the lack of clarity with required dosage [30] may further reduce the confidence of African populations in the safety of the vaccines [21]. Layered on top of this was the halting of the AstraZeneca vaccine in South Africa, which showed less protection against the new variant SARS-CoV-2 that can apparently evade key antibodies [21], and may have contributed to lower people's confidence in the vaccine efficacy. Health care workers are among the most trusted experts [31-33].

Intensive global efforts for continued physical distancing and isolation to curb the spread of new strains of the SARS-CoV-2 may intensify the use of social media as individuals try to remain connected while apart [34]. In a randomized controlled trial to understand the impact of social media in the United States, authors found that messages spread by nurses and doctors on social media led to a significant reduction in holiday travel and subsequent COVID-19 infections [14]. Therefore, identifying, understanding, and addressing how information sources affect vaccine acceptance [35], hesitancy and resistance [36] is potentially important to increase uptake of the vaccines

Therefore, this study was designed to, a) determine the proportions of SSA respondents that were dependent on the different sources of information (social media and mainstream media sources) for COVID-19 related information; b), profile individuals who use the mainstream media outlets (TV and radio, newspaper) to obtain COVID-19 related information by identifying the key socio-demographic, and health-related factors that are associated with the different information sources; and c), determine the sources of information about the COVID-19 pandemic among vaccine hesitant and resistant individuals across SSA countries as well as identify the association between sources of information and vaccine hesitancy. By identifying the distinguishing characteristics, public health officials may be better able to target a sub-population at greater risk of exposure to misinformation about COVID-19 vaccine. Findings will also offer a greater understanding of how public health officials can effectively tailor health behaviour messaging to align with the sociodemographic profiles of vaccine hesitant or resistant individuals, while also considering their consumption of COVID-19 information and the predominant sources. In addition, the study findings will help to provide steps on how social media may be used to improve health literacy and build public trust in vaccination.

\section{Materials And Methods}

\section{Ethics}

This self-administered web-based cross-sectional study was approved by the Humanities and Social Sciences Research Ethics Committee (approval\#: HSSREC 00002504/2021) of the University of KwaZulu-Natal, Durban, South Africa. The study adhered to the principles of the 1967 Helsinki declaration (as modified in Fortaleza 2013) for research involving human subjects. Prior to the study, an explanation detailing the nature and purpose of the study was provided to all participants using an online preamble. Informed consent was obtained from the participants who were required to answer either a 'yes' or 'no' to a question on whether they were willing to voluntarily participate in the survey. The confidentiality of participant responses was assured, 
and anonymity maintained. To ensure that only one response per respondent was included in the study, participants were instructed not to take part in the survey more than once, and during analysis we also restricted the data by IP address of the participants.

\section{Study population}

We purposively sampled only SSA respondents and a single population proportion formula by the WHO was used to determine the required sample size. Assuming a $20 \%$ attrition rate for a proportion of $50 \%$ of the population and using a desired precision of $2 \%$ and $5 \%$, significance level for a two-sided test to detect statistical differences between groups at $80 \%$ power, a sample size of 2502 was needed. Respondents were proportionately determined across the SSA countries. The study population consisted of adults aged 18 years and older from SSA countries which are shown in Fig. 1. Eligible participants were consenting English and French-speaking participants from SSA countries, at the time of this survey.

\section{Survey design}

The survey tool was a validated self-administered questionnaire initially developed and used for a similar study[37]. The survey tool was tested for the internal validity of the items, and the Cronbach's alpha coefficient score ranged from 0.70 and 0.74 , indicating satisfactory consistency[37]. The questionnaire was adapted with minor modifications to suit this study's objective and was made available in English and French languages to accommodate participants from the Anglophone and Francophone SSA countries. This was to increase the reach of the survey, one of the past study limitations $[26,27]$. Supplementary Table 1 shows a sample of the survey item. Moreover, a pilot study was conducted on 10 participants who were not included in the final study and were not part of the research group, to ensure clarity and understanding as well as to determine the duration for completing the questionnaire prior to dissemination..

\section{Data Collection Procedure}

The survey was web-based using online survey monkey (SurveyMonkey Inc, San Mateo, California, USA, www.surveymonkey.com) and was administered in both languages. An e-link to the survey was disseminated via emails and posted on social media platforms (Facebook and WhatsApp) and was available for four weeks (March 14 and May 16, 2021).

Participation in the study was voluntary without any incentive, inducement, or obligation from the researchers. Participants, who had access to the internet, were on the respective social media platforms and used them, may have participated. To avoid repeated responses, participants were instructed not to complete the survey if they had done so previously.

\section{Dependent variables}

The main outcome was derived from the question on how the participants get most of their information on COVID-19 vaccine. The participants responded 'yes' or 'no' to whether they obtained most of the information from the main stream media (Radio, Television, Newspaper), Social media (such as Facebook, WhatsApp, Twitter) and health care workers, and/or from family and friends.

\section{Exposure variables}


The exposure variables were the three COVID-19 vaccine indicators of the respondents. The vaccinated group were those who responded in affirmation (Yes) to the question on whether they have been vaccinated against COVID-19. Similar with previous study [38], those who responded 'not sure' or 'no' regarding being vaccinated against COVID-19 vaccine were asked if they were willing to be vaccinated when the vaccine becomes available in their home countries. The responses to the follow up question were used to derive the second and third outcome variables of 'vaccine hesitancy' and 'vaccine resistance' corresponding to those who responded with 'not sure' and the 'no' to the follow up question. In this study, vaccine acceptance refers to as a position ranging from passive acceptance to active demand [35], whereas hesitancy and resistance, respectively, are used to define positions where one is unsure about taking a vaccine and where one is absolutely against taking a vaccine, respectively [36].

\section{Independent variables}

The questionnaire included demographic data (age group, sex, country of origin, religion, marital status, educational level, employment status, occupational status), risk factors (smoking status, presence of preexiting condition including diabetes, lung disease, heart disease, hypertension, obesity, asthma and if they received past immunisations/vaccines. These constituted the independent variables.

\section{Statistical Analysis}

STATA/MP version 14 (Stata Corp, College Station, TX, USA) was used to perform all statistical analyses. In the descriptive statistics, categorical variables were presented as counts and percentages of each category. Proportion of respondents who used each of the sources of information was conducted using cross tabulation. Multinomial logistic regression analyses were used to examine the Covid-19 vaccination status on sources of information. As part of the multiple multinomial logistic regression analyses, a staged modelling technique was carried out.

As a process of multiple multinomial logistic regression modelling technique, demographic factors were first entered into the baseline multiple regression model with elimination process to remove statistically nonsignificant variables (Model 1). In the next stage, health indicators factors were examined with model 1 (Model 2). In the final model (model 3), the exposure variables sources of information were examined with those variables significant in models 1 and 2 . In the final model, we tested and reported any co-linearity. The odds ratios with $95 \%$ confidence intervals were calculated to assess the adjusted odds of independent.

\section{Results}

Data from 2572 SSA adults aged 18 years and above were collected and their socio-demographic characteristics are reported in Table 1 . These participants were mostly males (1390,54\%), aged 18-28years $(929,36.1 \%)$, not married $(1440,56.0 \%)$ and resided in West African countries $(1446,56.2 \%)$. About $80 \%$ of the respondents completed a bachelor or higher education degree and were employed in non-healthcare sectors. Of health indicators, there were few smokers $(177,6.9 \%)$ and people who reported that they had a pre-existing condition $(880,34.2 \%)$. 
Table 1

The socio-demographic characteristics of the study sample $(n=2572)$ and their sources of information

\begin{tabular}{|c|c|c|c|c|c|c|c|}
\hline Variables & All & Radio & TV & Newspaper & Facebook & HCW & Family/friends \\
\hline \multicolumn{8}{|l|}{ Demography } \\
\hline \multicolumn{8}{|l|}{ 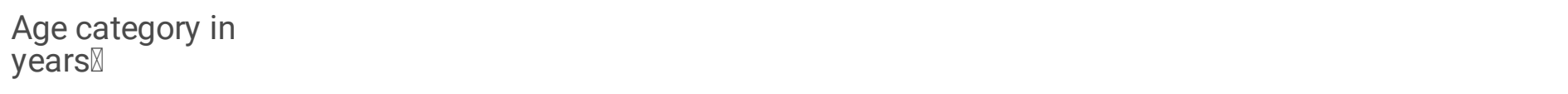 } \\
\hline $18-28$ & $\begin{array}{l}929 \\
(36.1)\end{array}$ & $\begin{array}{l}497 \\
(54.0)\end{array}$ & $\begin{array}{l}656 \\
(70.6)\end{array}$ & $347(37.4)$ & $\begin{array}{l}682 \\
(73.4)\end{array}$ & $\begin{array}{l}437 \\
(47.0)\end{array}$ & 461 (49.6) \\
\hline $29-38$ & $\begin{array}{l}720 \\
(28.0)\end{array}$ & $\begin{array}{l}415 \\
(57.6)\end{array}$ & $\begin{array}{l}532 \\
(73.9)\end{array}$ & $293(40.7)$ & $\begin{array}{l}523 \\
(72.6)\end{array}$ & $\begin{array}{l}363 \\
(50.4)\end{array}$ & $321(44.6)$ \\
\hline $39-48$ & $\begin{array}{l}502 \\
(19.5)\end{array}$ & $\begin{array}{l}293 \\
(58.4)\end{array}$ & $\begin{array}{l}390 \\
(77.7)\end{array}$ & $212(42.2)$ & $\begin{array}{l}364 \\
(72.5)\end{array}$ & $\begin{array}{l}271 \\
(54.0)\end{array}$ & $228(45.4)$ \\
\hline $49+$ & $\begin{array}{l}346 \\
(13.5)\end{array}$ & $\begin{array}{l}201 \\
(58.1)\end{array}$ & $\begin{array}{l}271 \\
(78.3)\end{array}$ & 177 (51.2) & $\begin{array}{l}265 \\
(76.6)\end{array}$ & $\begin{array}{l}178 \\
(51.4)\end{array}$ & $164(47.4)$ \\
\hline \multicolumn{8}{|l|}{ Sex } \\
\hline Males & $\begin{array}{l}1390 \\
(54.0)\end{array}$ & $\begin{array}{l}829 \\
(59.6)\end{array}$ & $\begin{array}{l}1047 \\
(75.3)\end{array}$ & $629(45.2)$ & $\begin{array}{l}1028 \\
(74.0)\end{array}$ & $\begin{array}{l}690 \\
(49.6)\end{array}$ & $623(44.8)$ \\
\hline Females & $\begin{array}{l}1182 \\
(46.0)\end{array}$ & $\begin{array}{l}620 \\
(52.4)\end{array}$ & $\begin{array}{l}850 \\
(71.9)\end{array}$ & $438(37.1)$ & $\begin{array}{l}851 \\
(72.0)\end{array}$ & $\begin{array}{l}599 \\
(50.7)\end{array}$ & $592(50.1)$ \\
\hline \multicolumn{8}{|l|}{ SSA region of origin $\Downarrow$} \\
\hline West Africa & $\begin{array}{l}1446 \\
(56.2)\end{array}$ & $\begin{array}{l}800 \\
(55.3)\end{array}$ & $\begin{array}{l}1054 \\
(72.9)\end{array}$ & $597(41.3)$ & $\begin{array}{l}1077 \\
(74.5)\end{array}$ & $\begin{array}{l}755 \\
(52.0)\end{array}$ & $668(46.2)$ \\
\hline East Africa & $\begin{array}{l}124 \\
(4.8)\end{array}$ & $\begin{array}{l}50 \\
(40.3)\end{array}$ & $\begin{array}{l}82 \\
(66.1)\end{array}$ & $48(38.7)$ & $96(77.4)$ & $\begin{array}{l}48 \\
(38.7)\end{array}$ & $45(36.3)$ \\
\hline Central Africa & $\begin{array}{l}314 \\
(12.2)\end{array}$ & $\begin{array}{l}184 \\
(58.6)\end{array}$ & $\begin{array}{l}251 \\
(79.9)\end{array}$ & $145(46.2)$ & $\begin{array}{l}225 \\
(71.7)\end{array}$ & $\begin{array}{l}176 \\
(56.1)\end{array}$ & $162(51.6)$ \\
\hline Southern Africa & $\begin{array}{l}667 \\
(25.9)\end{array}$ & $\begin{array}{l}409 \\
(61.3)\end{array}$ & $\begin{array}{l}500 \\
(75.0)\end{array}$ & $269(40.3)$ & $\begin{array}{l}472 \\
(70.8)\end{array}$ & $\begin{array}{l}303 \\
(45.4)\end{array}$ & 332 (49.8) \\
\hline \multicolumn{8}{|l|}{ Marital status } \\
\hline Married & $\begin{array}{l}1132 \\
(44.0)\end{array}$ & $\begin{array}{l}648 \\
(57.2)\end{array}$ & $\begin{array}{l}866 \\
(76.5)\end{array}$ & $472(41.7)$ & $\begin{array}{l}821 \\
(72.5)\end{array}$ & $\begin{array}{l}590 \\
(52.0)\end{array}$ & 505 (44.6) \\
\hline Not married§ & $\begin{array}{l}1440 \\
(56.0)\end{array}$ & $\begin{array}{l}801 \\
(55.6)\end{array}$ & $\begin{array}{l}1031 \\
(71.6)\end{array}$ & 595 (41.3) & $\begin{array}{l}1058 \\
(73.5)\end{array}$ & $\begin{array}{l}699 \\
(49.0)\end{array}$ & $710(49.3)$ \\
\hline \multicolumn{8}{|l|}{$\begin{array}{l}\text { Highest level of } \\
\text { education }\end{array}$} \\
\hline $\begin{array}{l}\text { Postgraduate degree } \\
\text { (Masters /PhD) }\end{array}$ & $\begin{array}{l}757 \\
(29.4)\end{array}$ & $\begin{array}{l}406 \\
(53.6)\end{array}$ & $\begin{array}{l}598 \\
(79.0)\end{array}$ & 335 (44.3) & $\begin{array}{l}567 \\
(74.9)\end{array}$ & $\begin{array}{l}378 \\
(49.9)\end{array}$ & $349(46.1)$ \\
\hline
\end{tabular}

HCW, health care workers. $\nabla=$ items have some missing responses; $\S=$ includes widowed, divorced and never married people 


\begin{tabular}{|c|c|c|c|c|c|c|c|}
\hline Variables & All & Radio & TV & Newspaper & Facebook & HCW & Family/friends \\
\hline Bachelor's degree & $\begin{array}{l}1309 \\
(50.9)\end{array}$ & $\begin{array}{l}750 \\
(57.3)\end{array}$ & $\begin{array}{l}955 \\
(73.0)\end{array}$ & $551(42.1)$ & $\begin{array}{l}969 \\
(74.0)\end{array}$ & $\begin{array}{l}707 \\
(54.0)\end{array}$ & $614(46.9)$ \\
\hline Secondary & $\begin{array}{l}448 \\
(17.4)\end{array}$ & $\begin{array}{l}262 \\
(58.5)\end{array}$ & $\begin{array}{l}312 \\
(69.6)\end{array}$ & $158(35.3)$ & $\begin{array}{l}314 \\
(70.1)\end{array}$ & $\begin{array}{l}181 \\
(40.4)\end{array}$ & $234(52.2)$ \\
\hline Primary or less & $\begin{array}{l}58 \\
(2.3)\end{array}$ & $\begin{array}{l}31 \\
(53.5)\end{array}$ & $\begin{array}{l}32 \\
(55.2)\end{array}$ & $23(39.7)$ & $29(50.0)$ & $\begin{array}{l}23 \\
(39.7)\end{array}$ & $18(31.0)$ \\
\hline \multicolumn{8}{|l|}{ Employment status } \\
\hline $\begin{array}{l}\text { Employed/self } \\
\text { employed }\end{array}$ & $\begin{array}{l}1890 \\
(73.5)\end{array}$ & $\begin{array}{l}1095 \\
(57.9)\end{array}$ & $\begin{array}{l}1428 \\
(75.6)\end{array}$ & $827(43.8)$ & $\begin{array}{l}1393 \\
(73.7)\end{array}$ & $\begin{array}{l}991 \\
(52.4)\end{array}$ & $872(46.1)$ \\
\hline Unemployed/retired & $\begin{array}{l}682 \\
(26.5)\end{array}$ & $\begin{array}{l}354 \\
(51.9)\end{array}$ & $\begin{array}{l}469 \\
(68.8)\end{array}$ & $240(35.2)$ & $\begin{array}{l}486 \\
(71.3)\end{array}$ & $\begin{array}{l}298 \\
(43.7)\end{array}$ & $343(50.3)$ \\
\hline \multicolumn{8}{|l|}{ Religion } \\
\hline Christianity & $\begin{array}{l}2301 \\
(89.5)\end{array}$ & $\begin{array}{l}1,324 \\
(57.5)\end{array}$ & $\begin{array}{l}1,736 \\
(75.4)\end{array}$ & $957(41.6)$ & $\begin{array}{l}1,699 \\
(73.8)\end{array}$ & $\begin{array}{l}1170 \\
(50.9)\end{array}$ & $1112(48.0)$ \\
\hline Others & $\begin{array}{l}271 \\
(10.5)\end{array}$ & $\begin{array}{l}125 \\
(46.1)\end{array}$ & $\begin{array}{l}161 \\
(59.4)\end{array}$ & $110(40.6)$ & $\begin{array}{l}180 \\
(66.4)\end{array}$ & $\begin{array}{l}119 \\
(43.9)\end{array}$ & $103(38.0)$ \\
\hline \multicolumn{8}{|l|}{ Occupation } \\
\hline $\begin{array}{l}\text { Non-healthcare } \\
\text { sector }\end{array}$ & $\begin{array}{l}1771 \\
(68.9)\end{array}$ & $\begin{array}{l}1017 \\
(57.4)\end{array}$ & $\begin{array}{l}1314 \\
(74.2)\end{array}$ & $760(42.9)$ & $\begin{array}{l}1301 \\
(73.5)\end{array}$ & $\begin{array}{l}801 \\
(45.0)\end{array}$ & 908 (51.3) \\
\hline Healthcare sector & $\begin{array}{l}801 \\
(31.1)\end{array}$ & $\begin{array}{l}432 \\
(53.9)\end{array}$ & $\begin{array}{l}583 \\
(72.8)\end{array}$ & 307 (38.3) & $\begin{array}{l}578 \\
(72.2)\end{array}$ & $\begin{array}{l}488 \\
(60.9)\end{array}$ & 307 (38.3) \\
\hline \multicolumn{8}{|l|}{ Health indicators } \\
\hline \multicolumn{8}{|l|}{ Smoking status } \\
\hline Ex-smoker & $\begin{array}{l}160 \\
(6.2)\end{array}$ & $\begin{array}{l}82 \\
(51.3)\end{array}$ & $\begin{array}{l}108 \\
(67.5)\end{array}$ & $66(41.3)$ & $\begin{array}{l}118 \\
(73.8)\end{array}$ & $\begin{array}{l}70 \\
(44.0)\end{array}$ & $63(39.4)$ \\
\hline Current smoker & $\begin{array}{l}177 \\
(6.9)\end{array}$ & $\begin{array}{l}114 \\
(64.4)\end{array}$ & $\begin{array}{l}132 \\
(74.6)\end{array}$ & $65(36.7)$ & $\begin{array}{l}133 \\
(75.1)\end{array}$ & $\begin{array}{l}75 \\
(42.4)\end{array}$ & $102(57.6)$ \\
\hline Non-smoker & $\begin{array}{l}2235 \\
(86.9)\end{array}$ & $\begin{array}{l}1,253 \\
(56.1)\end{array}$ & $\begin{array}{l}1657 \\
(74.1)\end{array}$ & $936(41.9)$ & $\begin{array}{l}1,628 \\
(72.8)\end{array}$ & $\begin{array}{l}1144 \\
(51.0)\end{array}$ & $1050(47.0)$ \\
\hline \multicolumn{8}{|l|}{$\begin{array}{l}\text { Any pre-existing } \\
\text { condition }\end{array}$} \\
\hline No & $\begin{array}{l}1692 \\
(65.8)\end{array}$ & $\begin{array}{l}1184 \\
(55.0)\end{array}$ & $\begin{array}{l}1568 \\
(72.9)\end{array}$ & $880(40.9)$ & $\begin{array}{l}1555 \\
(72.3)\end{array}$ & $\begin{array}{l}1056 \\
(49.0)\end{array}$ & $1008(46.9)$ \\
\hline Yes & $\begin{array}{l}880 \\
(34.2)\end{array}$ & $\begin{array}{l}265 \\
(63.0)\end{array}$ & $\begin{array}{l}329 \\
(78.2)\end{array}$ & $187(44.4)$ & $\begin{array}{l}324 \\
(77.0)\end{array}$ & $\begin{array}{l}233 \\
(55.0)\end{array}$ & 207 (49.2) \\
\hline
\end{tabular}

HCW, health care workers. $\mathrm{X}$ = items have some missing responses; $\S=$ includes widowed, divorced and never married people 


\begin{tabular}{|c|c|c|c|c|c|c|c|}
\hline Variables & All & Radio & TV & Newspaper & Facebook & HCW & Family/friends \\
\hline \multicolumn{8}{|c|}{$\begin{array}{l}\text { History of previous } \\
\text { vaccination }\end{array}$} \\
\hline No & $\begin{array}{l}1692 \\
(65.8)\end{array}$ & $\begin{array}{l}910 \\
(53.8)\end{array}$ & $\begin{array}{l}1,229 \\
(72.6)\end{array}$ & $661(39.1)$ & $\begin{array}{l}1,237 \\
(73.1)\end{array}$ & $\begin{array}{l}803 \\
(47.0)\end{array}$ & 793 (46.9) \\
\hline Yes & $\begin{array}{l}880 \\
(34.2)\end{array}$ & $\begin{array}{l}539 \\
(61.3)\end{array}$ & $\begin{array}{l}668 \\
(75.9)\end{array}$ & $406(46.1)$ & $\begin{array}{l}642 \\
(72.9)\end{array}$ & $\begin{array}{l}486 \\
(55.0)\end{array}$ & $422(47.9)$ \\
\hline
\end{tabular}

\section{Insert Table 1}

Television and Facebook were the main sources of information for majority of the respondents in this study during the pandemic, while very few people relied on the newspaper for such information (Table 1). This was consistent across regions, age groups and gender. More than half of the Central African respondents reported that they sought for COVID-19 related information from health care workers (HCWs), whereas East African respondents relied less on this source of information. Fifty-five percent of those with pre-existing health condition and those that had previous vaccination, reported that they relied on HCWs for COVID-19 related information.

\section{Prevalence of vaccine acceptance, hesitance, and resistance by the information sources}

Figure 1 displays the proportions in the prevalence of vaccination, resistance, and hesitancy for the different sources of information used by the respondents in this study. Overall, the prevalence of vaccine hesitancy was highest among those that received COVID-19 related information from television (73.0\%) and resistance was highest among users of social media ( $84.5 \%$ ) during the pandemic. However, the lowest prevalence of vaccine hesitancy and resistance was recorded among newspapers readers ( $36.7 \%$ and $42.5 \%$, respectively). A total of $63.3 \%$ of social media and $70.3 \%$ of mainstream media listeners were vaccinated with the lowest rates found among those that received information from friends and family members (40.3\%) where more than half of them were vaccine hesitant (53.5\%), at the time of this study. As can be seen from Fig. 1, among those who relied on HCWs for information during the pandemic, vaccination rate was high while hesitancy rate was low.

\section{Insert Fig. 1}

\section{Socio-demographic, and health indicators associated with COVID-19 related information sources}

The full set of findings from the multinomial logistic regression analyses showing the characteristics of those that relied on the various sources of information during the pandemic, after adjusting for the potential cofounders are presented in Tables 2. In this sample, reliance on the mainstream media for information during the pandemic was associated with region of origin (Central and Southern African respondents), whereas reliance on social media was associated with educational level and religion. Reliance on information from 
social media decreased with lower education but significant only among those with primary or no education (AORs $=0.36,95 \% \mathrm{Cl}=0.20,0.62$ ). The odds were also significantly lower among non-Christians compared with Christians. 
Table 2

Adjusted odd ratios of factors associated with the information sources for COVID-19.

\begin{tabular}{|c|c|c|c|c|c|c|}
\hline Variables & Radio & Television & Newspaper & $\begin{array}{l}\text { Social } \\
\text { media }\end{array}$ & HCW & Family/Friends \\
\hline Demography & $\begin{array}{l}\text { AORs } \\
{[95 \% \mathrm{Cl}]}\end{array}$ & $\begin{array}{l}\text { AORs } \\
{[95 \% \mathrm{Cl}]}\end{array}$ & $\begin{array}{l}\text { AORs [95\% } \\
\mathrm{Cl}]\end{array}$ & $\begin{array}{l}\text { AORs } \\
{[95 \% \mathrm{Cl}]}\end{array}$ & $\begin{array}{l}\text { AORs } \\
{[95 \% \mathrm{Cl}]}\end{array}$ & AORs [95\% Cl] \\
\hline \multicolumn{7}{|l|}{ Sex } \\
\hline Males & Reference & Reference & Reference & & & Reference \\
\hline Females & $\begin{array}{l}0.72 \\
{[0.81} \\
0.84]\end{array}$ & $\begin{array}{l}0.81 \\
{[0.68} \\
0.98]\end{array}$ & $\begin{array}{l}0.73[0.62, \\
0.86]\end{array}$ & - & - & $\begin{array}{l}1.23[1.05 \\
1.45]\end{array}$ \\
\hline \multicolumn{7}{|l|}{$\begin{array}{l}\text { SSA region of } \\
\text { origin }\end{array}$} \\
\hline West Africa & Reference & Reference & Reference & Reference & Reference & Reference \\
\hline East Africa & $\begin{array}{l}0.53 \\
{[0.37} \\
0.78]\end{array}$ & $\begin{array}{l}0.74 \\
{[0.50} \\
1.10]\end{array}$ & $\begin{array}{l}0.88[0.60 \\
1.29]\end{array}$ & $\begin{array}{l}1.18[0.76, \\
1.83]\end{array}$ & $\begin{array}{l}0.56 \\
{[0.38} \\
0.82]\end{array}$ & $\begin{array}{l}0.66[0.45, \\
0.97]\end{array}$ \\
\hline Central Africa & $\begin{array}{l}1.16 \\
{[0.90} \\
1.50]\end{array}$ & $\begin{array}{l}1.69 \\
{[1.24} \\
2.29]\end{array}$ & $\begin{array}{l}1.20[0.93 \\
1.54]\end{array}$ & $\begin{array}{l}0.92[0.70, \\
1.22]\end{array}$ & $\begin{array}{l}1.37 \\
{[1.07} \\
1.77]\end{array}$ & $\begin{array}{l}1.12[0.87, \\
1.44]\end{array}$ \\
\hline Southern Africa & $\begin{array}{l}1.49 \\
{[1.22} \\
1.81]\end{array}$ & $\begin{array}{l}1.44 \\
{[1.14} \\
1.81]\end{array}$ & $\begin{array}{l}1.11[0.91, \\
1.36]\end{array}$ & $\begin{array}{l}0.89 \\
{[0.72} \\
1.11]\end{array}$ & $\begin{array}{l}0.89 \\
{[0.73} \\
1.08]\end{array}$ & $\begin{array}{l}1.03[0.84 \\
1.27]\end{array}$ \\
\hline \multicolumn{7}{|l|}{$\begin{array}{l}\text { Highest level of } \\
\text { education }\end{array}$} \\
\hline $\begin{array}{l}\text { Postgraduate } \\
\text { degree (Masters } \\
\text { /PhD) }\end{array}$ & & Reference & Reference & Reference & Reference & Reference \\
\hline Bachelor's degree & & $\begin{array}{l}0.71 \\
{[0.57} \\
0.88]^{\prime}\end{array}$ & $\begin{array}{l}0.97[0.81 \\
1.17]\end{array}$ & $\begin{array}{l}0.95 \\
{[0.77} \\
1.17]\end{array}$ & $\begin{array}{l}1.20 \\
{[1.00} \\
1.45]\end{array}$ & $\begin{array}{l}1.01[0.84 \\
1.21]\end{array}$ \\
\hline Secondary & & $\begin{array}{l}0.53 \\
{[0.40} \\
0.70]\end{array}$ & $\begin{array}{l}0.73[0.55 \\
0.96]\end{array}$ & $\begin{array}{l}0.82 \\
{[0.62,} \\
1.08]\end{array}$ & $\begin{array}{l}0.86 \\
{[0.67} \\
1.11]\end{array}$ & $\begin{array}{l}0.96[0.74 \\
1.24]\end{array}$ \\
\hline Primary or less & & $\begin{array}{l}0.34 \\
{[0.19} \\
0.61]\end{array}$ & $\begin{array}{l}0.96[0.54 \\
1.69]\end{array}$ & $\begin{array}{l}0.36 \\
{[0.20} \\
0.62]\end{array}$ & $\begin{array}{l}0.83 \\
{[0.47} \\
1.46]\end{array}$ & $\begin{array}{l}0.44[0.25 \\
0.80]\end{array}$ \\
\hline \multicolumn{7}{|l|}{ Employment status } \\
\hline $\begin{array}{l}\text { Employed/self } \\
\text { employed }\end{array}$ & Reference & & Reference & & & \\
\hline
\end{tabular}

Confidence intervals $(\mathrm{Cl})$ that does not include 1.00 are significant variables. 


\begin{tabular}{|c|c|c|c|c|c|c|}
\hline Variables & Radio & Television & Newspaper & $\begin{array}{l}\text { Social } \\
\text { media }\end{array}$ & HCW & Family/Friends \\
\hline Unemployed/retired & $\begin{array}{l}0.72 \\
{[0.60} \\
0.88]\end{array}$ & & $\begin{array}{l}0.72[0.59, \\
0.89]\end{array}$ & & & \\
\hline \multicolumn{7}{|l|}{ Religion } \\
\hline Christianity & Reference & Reference & & Reference & & \\
\hline Others & $\begin{array}{l}0.57 \\
{[0.44} \\
0.74]\end{array}$ & $\begin{array}{l}0.45 \\
{[0.34} \\
0.59]\end{array}$ & & $\begin{array}{l}0.74 \\
{[0.56} \\
0.97]\end{array}$ & & $\begin{array}{l}0.65[0.50 \\
0.85]\end{array}$ \\
\hline \multicolumn{7}{|l|}{ Occupation } \\
\hline $\begin{array}{l}\text { Non-healthcare } \\
\text { sector }\end{array}$ & Reference & & Reference & & Reference & Reference \\
\hline Healthcare sector & $\begin{array}{l}0.82 \\
{[0.69} \\
0.99]\end{array}$ & & $\begin{array}{l}0.71[0.59, \\
0.86]\end{array}$ & & $\begin{array}{l}1.81 \\
{[1.51} \\
2.17]\end{array}$ & $\begin{array}{l}0.58[0.48 \\
0.69]\end{array}$ \\
\hline \multicolumn{7}{|l|}{ Smoking status } \\
\hline Ex-smoker & & & & & & Reference \\
\hline Current smoker & & & & & & $\begin{array}{l}1.97[1.26 \\
3.10]\end{array}$ \\
\hline Non-smoker & & & & & & $\begin{array}{l}1.35[0.96, \\
1.89]\end{array}$ \\
\hline
\end{tabular}

\section{Insert Table 2}

Respondents who worked in health sectors, and those who were from Central Africa were more likely to rely on information from HCWs during the pandemic as compared with those who worked in non-health care sectors and people who were from the West African countries. Compared with males, females were less likely to listen to the radio, watch $\mathrm{TV}$ and read newspapers but more likely to rely on friends and family $(\mathrm{AOR}=1.23,95 \% \mathrm{Cl}=$ $1.05,1.45)$, for information regarding COVID-19 during the pandemic. Current smokers were also more likely to rely on friends and family $(A O R=1.97,95 \% \mathrm{Cl}=1.26,3.10)$ while those with primary or no education as well as non-Christians were less likely to rely on social media for information during the pandemic.

\section{Associations between COVID-19 vaccine hesitancy, resistance, and sources of information}

Figure 2 presents a forest plot of the AORs for the association between vaccine hesitancy, resistance, and the information sources during the pandemic in SSA. For this model, we added the COVID related variables of vaccination, hesitancy and resistance to the variables used on Table 2 . There were no statistically significant associations between listening to radio and obtaining information from HCWs and any of the COVID related variables of hesitancy and resistance. Those who watched television (AORs $=1.54,95 \% \mathrm{Cl}=1.11,2.14$ ) and 
people who relied on their families and friends (AORs $=1.44,95 \% \mathrm{Cl}=1.08,1.93)$ for information about COVID19 were more likely to be resistant, and those who read newspaper were less likely to be resistant (AORs $=0.69$, $95 \% \mathrm{Cl}=0.52,0.93)$ compared with those who were vaccinated.

Respondents who relied on social media for information during the pandemic, were more likely to be hesitant and resistant with the odds increasing by $1.46(95 \% \mathrm{Cl}=1.15,1.85)$ and $2.93(95 \% \mathrm{Cl}=2.10,4.09)$ times, respectively, compared with the vaccinated group.

\section{Insert Fig. 2}

\section{Discussion}

This study was undertaken to determine the role of different information sources on COVID-19 vaccine acceptance, hesitancy, and resistance in Sub-Saharan Africa. Consistent across age groups, gender and regions, Television and Facebook were the main media platforms used by most respondents for information related to COVID-19 during the lockdown. Respondents from Central and Southern Africa were more reliant on information obtained from watching TV. Smokers, and females were more likely to rely on family and friends for COVID-19 related information, but less likely to rely on mainstream media (such as TV) than their male counterparts. There was a lower likelihood for non-Christians and those with lower education to rely on social media for information during the lockdown. Of the information sources, reliance on social media showed the strongest association with COVID vaccine hesitancy and resistance. The results of the study showed that after adjusting for potential confounders, information sources played a significant role in vaccine hesitancy and resistance of SSA. Those who rely on TV and information from family/friends were more likely to resist COVID vaccine when compared with those who were vaccinated. However, social media use played the highest role in vaccine hesitancy and resistance as the users were almost twice more likely to hesitate and thrice more likely to resist taking the vaccination.

The fact that social media such as Facebook was one of the main sources of information among these respondents during the lockdown, suggests their wide utilisation among sub-Saharan regions. This finding is consistent with a Facebook IQ survey, which revealed that more than 95 million people in sub-Saharan Africa access Facebook, with $97 \%$ of these doing so on handheld and mobile devices, each month. It is therefore possible that Television and Facebook can be used to convey reliable, science-based information about COVID-19 vaccines and future pandemics to the millions of SSA people.

Listening to the radio and obtaining information from $\mathrm{HCWs}$ had a positive influence on intent towards vaccination because it reduced their likelihood of being resistant and hesitant towards COVID-19 vaccination. The negative influence of TV and social media use on COVID-19 vaccination reported in this study was not surprising as some emerging anti-vaccine Television and social media campaigns are responsible for generating and perpetuating vaccine hesitancy and resistance. The high prevalence of inaccurate and negative information on social media regarding COVID-19 may predict a greater likelihood of negative vaccine intent in this case as well $[39,40]$. In addition, social media is also generally unregulated and has enabled people with anti-vaccine beliefs to generate and disseminate information freely [41]. The findings of this study are consistent with a previous study which found that, relative to social media and internet, there was a positive association between reliance on traditional news sources and intention to uptake a COVID-19 vaccine 
in the United States [42]. Another previous work also highlighted the role of negative information on social media in shaping individual perceptions regarding human papillomavirus (HPV) vaccination intent [43].

Most respondents with pre-existing medical conditions or prior history of vaccinations relied on health care workers (HCWs) for COVID-19 related information. This is an important finding and suggests that HCWs have a better understanding of the experiences of COVID-19 information and they can provide essential care and information in future pandemics. This assertion is supported by a recent study which showed that HCWs are essential front lines, working to ensure the health of older adults and those with chronic conditions or disabilities during the COVID-19 pandemic[44]. A study has also indicated that people rated health information from doctors and other health works as highly reliable reaching $97 \%$ reliable for doctors [45].

The high vaccination and low hesitancy rates reported among respondents who relied on HCWs for information was in agreement with a previous report which showed that HCWs have adequate information on vaccines and have the ability and confidence to communicate such information effectively [46]. These factors are important and can influence vaccine acceptance and uptake. However, recent literature has also warned that one of the impediments in dealing with anti-vaccine social media messages is that HCWs have inadequate capacity to respond to these messages [47]. In addition, hesitancy was low among those who received COVID-19 related information from HCWs (Fig. 1). This finding further supports the idea that HCWs, across board can positively influence the use of vaccines and thus health workers have the potential to impact on COVID-19 vaccination in SSA.

The finding that respondents from Central and Southern Africa relied on the mainstream media for COVID-19 related information, particularly TV watching, and this was associated with vaccine resistance could in part, be related to the nature of lockdowns in different sub-Saharan countries. For instance, South Africa went into Level 5 (hard lockdown) quite early in the pandemic (March 2020) and residents were mostly confined to their homes, watching TV [48]. Reliance on COVID-19 information from social media platforms decreased with lower levels of education indicating that educational levels play a key role in obtaining COVID-19 information from social media. Kollamparambil and Oyenubi [48] reported that education-related inequalities were visible in the use of COVID-19 preventive measures in South Africa. Also, compared to the Christian majority, nonChristian respondents were less likely to rely on information from social media.

Females were less likely to listen to radio, watch TV and read newspaper but more likely to rely on friends and family and this increased their likelihood of vaccine hesitancy. This may suggest that women expressed interest in COVID-19 issues with their friends and family (leaving very little room for individual proactive decision-making) while men were significantly more likely than women to get such information from the radio, TV and newspapers. The study also showed that there were differences in behavior: being less educated or non-Christians, was associated with a less chance of relying on social media platforms for information during the pandemic. For those who were more likely to be resistant (such as those who watched TV and those who relied on their families and friends for information), additional vaccine promotional efforts would be required.

\section{Limitations and strengths}


There are some limitations that should be considered when interpreting the findings of this study. First, this was a cross-sectional study and as such we cannot determine causation. Second, similar to previous studies conducted during COVID-19 in SSA[27, 49-51], we utilized an internet-based methodology which was the only reliable means to disseminate information at the time of this study. The survey was distributed electronically using social media platforms and emails because it was difficult to physically access some respondents in some places due to the protective measures still in place at the time of study. This method of soliciting respondents may have inadvertently excluded some potential participants whose opinion may have differed, such as those without internet access, and people living in rural areas, where internet penetration remains relatively low[52]. Third, the survey was presented in English and French and thus inadvertently excluding nonEnglish and non-French speaking countries in SSA from participating. Fourth, although the study showed satisfactory internal validity, its generalization or transferability to all SSA countries may be limited.

Notwithstanding these limitations, this was the first study from the SSA region to provide insight into some of the impacts of information sources on the acceptance of COVID-19 vaccines which has been a worry to the international community. Although this topic is commonplace as reliance on online information sources is expected to happen during pandemics, no study has demonstrated these impacts of these sources of information on COVID vaccination in the way the present study did including the use of a robust analysis to control for potential confounders during the analysis and reduce the possibility of a bias. This makes our study a unique one since it provided the first documented evidence from SSA showing the impacts of the lockdown on the behaviour of ordinary citizens.

\section{Implications of our findings}

This study provides an understanding of how the information Africans are exposed to influence their attitude towards COVID-19 vaccine. Our focus on COVID-19 vaccine hesitancy and resistance is important because to stem the pandemic, enough people need to get vaccinated against COVID-19[6]. The findings are important because people's negative attitudes toward vaccination in general, and their hesitancy or resistance towards COVID-19 vaccine, is a growing public health problem. This study provides insight into how the various media outlets commonly used by the respondents living in different SSAs regions to obtain COVID-19 related information affects their attitude towards vaccine uptake, which underlines the importance of media exposure. These media sources can be used to improve vaccine literacy across the region[53].

In addition, this study contributes to our understanding of the interplay between SSA regions and media exposure during the pandemic. For example, the study found greater reliance on the mainstream media for COVID-19 related information among those from Central and Southern Africa, which then had negative influence on vaccine uptake. This insight has important practical implications by informing us about the dynamics of individuals' attitudes, which helps in understanding the underlying factors that influence acceptance of vaccination during a pandemic. Knowledge gained from this study will help public health and health promotion officers in various SSA countries to design more effective communications and interventions.

\section{Conclusions}


COVID-19 clearly exposes the fact that more research and investment are needed to improve the availability of reliable healthcare information, to protect people from misinformation, and to empower people to tell the difference. The trajectory of misinformation over the past 20 years - from vaccine hesitancy to previous infectious diseases to COVID-19 - shows that if we do not act, the 'infodemic' of the next public health emergency may be worse than the current COVID infodemic. Meanwhile, it should be noted that failure to access and apply reliable healthcare information, whether for the public or for health workers, is and always has been a major cause of avoidable non-COVID deaths across the life course and across all areas of health. Further studies including other are needed

\section{Abbreviations}

COVID-19

Coronavirus disease

SSA

sub Saharan Africa

OR

Odds ratio

AOR

Adjusted odds ratio

$\mathrm{Cl}$

Confidence interval

TV

Television

HCW

Health care worker

WHO

World Health Organization

\section{Declarations}

\section{Ethics approval and consent to participate}

The study was conducted in accordance with the Declaration of Helsinki and approved by the Humanities and Social Sciences Research Ethics Committee (approval \#: HSSREC 00002504/2021) of the University of KwaZulu-Natal, Durban, South Africa for studies involving humans. Informed consent was obtained from all participants involved in the study.

\section{Consent for publication}

Not applicable

\section{Availability of data and materials}


The dataset supporting the conclusions of this article is included within the article (and its additional files). Data is also available on request from the corresponding author OUL.

\section{Competing interests}

The authors declare that they have no competing interests

\section{Funding}

This research did not receive any specific grant from funding agencies in the public, commercial, or not-forprofit sectors.

\section{Authors' contributions}

All authors were involved in the conceptualization of the study; KEA, ULO., and KPM performed the methodology; Software, KEA, ULO; Validation, TI, RO, EE, BNE, OA, KPM, EKA, MC and TC; Formal Analysis, KEA, and ULO; Investigation, all authors; Resources, all authors; Data Curation, KEA, ON, and ULO; Writing - Original Draft Preparation, PCG, GO, RO; EE, ULO, EA; Writing - Review \& Editing, KPM, GO, OA, EA, KEA, KPM, RL, DDC, and MC; Visualization, KPM, and KEA; Supervision, KA, ULO, TI, BE, KPM; Project Administration, KA, ULO and PCG. All authors have read and agreed to the published version of the manuscript.

\section{Acknowledgement}

None

\section{References}

1. Semo B-w, Frissa SM: The mental health impact of the COVID-19 pandemic: Implications for sub-Saharan Africa. Psychology Research and Behavior Management 2020, 13:713.

2. Kassema JJ: COVID-19 outbreak: is it a health crisis or economic crisis or both? Case of african counties. Case of African Counties (March 23, 2020) 2020.

3. Ataguba JE: COVID-19 pandemic, a war to be won: understanding its economic implications for Africa. In.: Springer; 2020.

4. WHO: African countries face high risk of COVID-19 resurgence. In. Edited by Boakye-Agyemang C, OKA S: World Health Organization Africa; 2021.

5. WHO: Africa on track to control COVID-19 pandemic in 2022. In. Edited by Dalal M. Brazzaville: WHO Africa; 2022.

6. Mellissa Q: Fauci warns "unlikely" to reach Herd Immunity if too Many Refuse Vaccine. In.: CBS News; 2020.

7. Basak P, Abir T, Al Mamun A, Zainol NR, Khanam M, Haque MR, Milton AH, Agho KE: A Global Study on the Correlates of Gross Domestic Product (GDP) and COVID-19 Vaccine Distribution. Vaccines 2022, 10(2):266. 
8. Losada-Baltar A, Jiménez-Gonzalo L, Gallego-Alberto L, Pedroso-Chaparro MdS, Fernandes-Pires J, Márquez-González M: "We are staying at home." Association of self-perceptions of aging, personal and family resources, and loneliness with psychological distress during the lock-down period of COVID-19. The Journals of Gerontology: Series B 2021, 76(2):e10-e16.

9. Hu G, Qiu W: From guidance to practice: Promoting risk communication and community engagement for prevention and control of coronavirus disease (COVID-19) outbreak in China. Journal of Evidence-Based Medicine 2020, 13(2):168-172.

10. Scheres J, Kuszewski K: The Ten Threats to Global Health in 2018 and 2019. A welcome and informative communication of WHO to everybody. Zeszyty Naukowe Ochrony Zdrowia Zdrowie Publiczne i Zarzadzanie 2019, 17(1):2-8.

11. Tustin JL, Crowcroft NS, Gesink D, Johnson I, Keelan J, Lachapelle B: User-driven comments on a Facebook advertisement recruiting Canadian parents in a study on immunization: content analysis. JMIR public health and surveillance 2018, 4(3):e10090.

12. Wilson SL, Wiysonge C: Social media and vaccine hesitancy. BMJ Global Health 2020, 5(10):e004206.

13. Wang P-W, Ahorsu DK, Lin C-Y, Chen I-H, Yen C-F, Kuo Y-J, Griffiths MD, Pakpour AH: Motivation to Have COVID-19 Vaccination Explained Using an Extended Protection Motivation Theory among University Students in China: The Role of Information Sources. Vaccines 2021, 9(4):380.

14. Breza E, Stanford FC, Alsan M, Alsan B, Banerjee A, Chandrasekhar AG, Eichmeyer S, Glushko T, Goldsmith-Pinkham $\mathrm{P}$, Holland K: Effects of a large-scale social media advertising campaign on holiday travel and COVID-19 infections: a cluster randomized controlled trial. Nature medicine 2021, 27(9):16221628.

15. Razai MS, Osama T, McKechnie DGJ, Majeed A: Covid-19 vaccine hesitancy among ethnic minority groups. BMJ 2021, 372:n513.

16. Robertson E, Reeve KS, Niedzwiedz CL, Moore J, Blake M, Green M, Katikireddi SV, Benzeval MJ: Predictors of COVID-19 vaccine hesitancy in the UK household longitudinal study. Brain, Behavior, and Immunity 2021, 94:41-50.

17. Anderson J: America has a history of medically abusing Black people. No wonder many are wary of COVID-19 vaccines. In: USA Today. 2021.

18. Nyhan B, Reifler J, Richey S, Freed GL: Effective messages in vaccine promotion: a randomized trial. Pediatrics 2014, 133(4):e835-e842.

19. Faasse $K$, Chatman CJ, Martin LR: A comparison of language use in pro-and anti-vaccination comments in response to a high profile Facebook post. Vaccine 2016, 34(47):5808-5814.

20. Reno C, Maietti E, Di Valerio Z, Montalti M, Fantini MP, Gori D: Vaccine Hesitancy towards COVID-19 Vaccination: Investigating the Role of Information Sources through a Mediation Analysis. Infectious Disease Reports 2021, 13(3):712-723.

21. Al-Shamsi HO, Alhazzani W, Alhuraiji A, Coomes EA, Chemaly RF, Almuhanna M, Wolff RA, Ibrahim NK, Chua ML, Hotte SJ: A practical approach to the management of cancer patients during the novel coronavirus disease 2019 (COVID-19) pandemic: an international collaborative group. The oncologist 2020, 25(6):e936. 
22. Puri N, Coomes EA, Haghbayan H, Gunaratne K: Social media and vaccine hesitancy: new updates for the era of COVID-19 and globalized infectious diseases. Human Vaccines \& Immunotherapeutics 2020:1-8.

23. Meleo-Erwin Z, Basch C, MacLean SA, Scheibner C, Cadorett V: “To each his own”: Discussions of vaccine decision-making in top parenting blogs. Human vaccines \& immunotherapeutics 2017, 13(8):1895-1901.

24. Daley MF, Glanz JM: Using social media to increase vaccine acceptance. Academic Pediatrics 2021, 21(4):S32-S33.

25. Donovan J: Social-media companies must flatten the curve of misinformation. Nature 2020.

26. Osuagwu UL, Miner CA, Bhattarai D, Mashige KP, Oloruntoba R, Abu EK, Ekpenyong B, Chikasirimobi TG, Goson PC, Ovenseri-Ogbomo GO: Misinformation about COVID-19 in sub-saharan africa: Evidence from a cross-sectional survey. Health security 2021, 19(1):44-56.

27. Ovenseri-Ogbomo G, Ishaya T, Osuagwu UL, Abu EK, Nwaeze O, Oloruntoba R, Ekpenyong B, Mashige KP, Chikasirimobi T, Langsi $\mathrm{R}$ et al: Factors associated with the myth about $5 \mathrm{G}$ network during COVID-19 pandemic in sub-Saharan Africa. Journal of Global Health Reports 2020, 4:1-13.

28. Wise J: Covid-19: European countries suspend use of Oxford-AstraZeneca vaccine after reports of blood clots. In.: British Medical Journal Publishing Group; 2021.

29. Shimabukuro TT, Cole M, Su JR: Reports of anaphylaxis after receipt of mRNA COVID-19 vaccines in the US-December 14, 2020-January 18, 2021. Jama 2021, 325(11):1101-1102.

30. Wise J: Covid-19: People who have had infection might only need one dose of mRNA vaccine. BMJ 2021, 372:n308.

31. Altman D: Why Doctors and Nurses Can Be Vital Vaccine Messengers. Kaiser Family Foundation, 2021. In.

32. Liz H, Ashley K, Lunna L, Audrey K, Grace S, Mollyann B: KFF COVID-19 Vaccine Monitor: January 2021. In: Coronavirus. Kaiser Family Foundation (KFF); 2021.

33. G MNdR: Doctors plead with Americans to take the virus surge 11 seriously. In: The New York Times. New York; 2020.

34. Limaye RJ, Sauer M, Ali J, Bernstein J, Wahl B, Barnhill A, Labrique A: Building trust while influencing online COVID-19 content in the social media world. The Lancet Digital Health 2020, 2(6):e277-e278.

35. Habersaat KB, Jackson C: Understanding vaccine acceptance and demand-and ways to increase them. Bundesgesundheitsblatt-Gesundheitsforschung-Gesundheitsschutz 2020, 63(1):32-39.

36. Larson HJ, Jarrett C, Eckersberger E, Smith DM, Paterson P: Understanding vaccine hesitancy around vaccines and vaccination from a global perspective: a systematic review of published literature, 20072012. Vaccine 2014, 32(19):2150-2159.

37. Biasio LR, Bonaccorsi G, Lorini C, Pecorelli S: Assessing COVID-19 vaccine literacy: a preliminary online survey. Human Vaccines \& Immunotherapeutics 2021, 17(5):1304-1312.

38. Murphy J, Vallières F, Bentall RP, Shevlin M, McBride O, Hartman TK, McKay R, Bennett K, Mason L, Gibson-Miller J et al: Psychological characteristics associated with COVID-19 vaccine hesitancy and resistance in Ireland and the United Kingdom. Nature Communications 2021, 12(1):29.

39. Fridman I, Lucas N, Henke D, Zigler CK: Association between public knowledge about COVID-19, trust in information sources, and adherence to social distancing: cross-sectional survey. JMIR public health and surveillance 2020, 6(3):e22060. 
40. Li HO-Y, Bailey A, Huynh D, Chan J: YouTube as a source of information on COVID-19: a pandemic of misinformation? BMJ global health 2020, 5(5):e002604.

41. Stein RA: The golden age of anti-vaccine conspiracies. Germs 2017, 7(4):168.

42. Piltch-Loeb R, Savoia E, Goldberg B, Hughes B, Verhey T, Kayyem J, Miller-ldriss C, Testa M: Examining the effect of information channel on COVID-19 vaccine acceptance. Plos one 2021, 16(5):e0251095.

43. Margolis MA, Brewer NT, Shah PD, Calo WA, Gilkey MB: Stories about HPV vaccine in social media, traditional media, and conversations. Preventive medicine 2019, 118:251-256.

44. Sterling MR, Tseng E, Poon A, Cho J, Avgar AC, Kern LM, Ankuda CK, Dell N: Experiences of home health care workers in New York City during the coronavirus disease 2019 pandemic: a qualitative analysis. JAMA internal medicine 2020, 180(11):1453-1459.

45. Worsley A: Perceived reliability of sources of health information. Health Education Research 1989, 4(3):367-376.

46. Falagas ME, Zarkadoulia E: Factors associated with suboptimal compliance to vaccinations in children in developed countries: a systematic review. Current medical research and opinion 2008, 24(6):1719-1741.

47. Goldstein S, MacDonald NE, Guirguis S: Health communication and vaccine hesitancy. Vaccine 2015, 33(34):4212-4214.

48. Kollamparambil U, Oyenubi A: Behavioural response to the Covid-19 pandemic in South Africa. PLOS ONE 2021, 16(4):e0250269.

49. Abir T, Osuagwu UL, Nur AYDM, Mamun AA, Kakon K, Salamah AA, Zainol NR, Khanam M, Agho KE: Internet Use Impact on Physical Health during COVID-19 Lockdown in Bangladesh: A Web-Based CrossSectional Study. Int J Environ Res Public Health 2021, 18(20).

50. Abu EK, Oloruntoba R, Osuagwu UL, Bhattarai D, Miner CA, Goson PC, Langsi R, Nwaeze O, Chikasirimobi TG, Ovenseri-Ogbomo GO et al: Risk perception of COVID-19 among sub-Sahara Africans: a web-based comparative survey of local and diaspora residents. BMC Public Health 2021, 21(1):1562.

51. Mashige KP, Osuagwu UL, Ulagnathan S, Ekpenyong BN, Abu EK, Goson PC, Langsi R, Nwaeze O, Timothy CG, Charwe DD: Economic, Health and Physical Impacts of COVID-19 Pandemic in Sub-Saharan African Regions: A Cross Sectional Survey. Risk Management and Healthcare Policy 2021, 14:4799.

52. Hjort J, Poulsen J: The arrival of fast internet and employment in Africa. American Economic Review 2019, 109(3):1032-1079.

53. Pugliese-Garcia M, Heyerdahl LW, Mwamba C, Nkwemu S, Chilengi R, Demolis R, Guillermet E, Sharma A: Factors influencing vaccine acceptance and hesitancy in three informal settlements in Lusaka, Zambia. Vaccine 2018, 36(37):5617-5624.

\section{Figures}




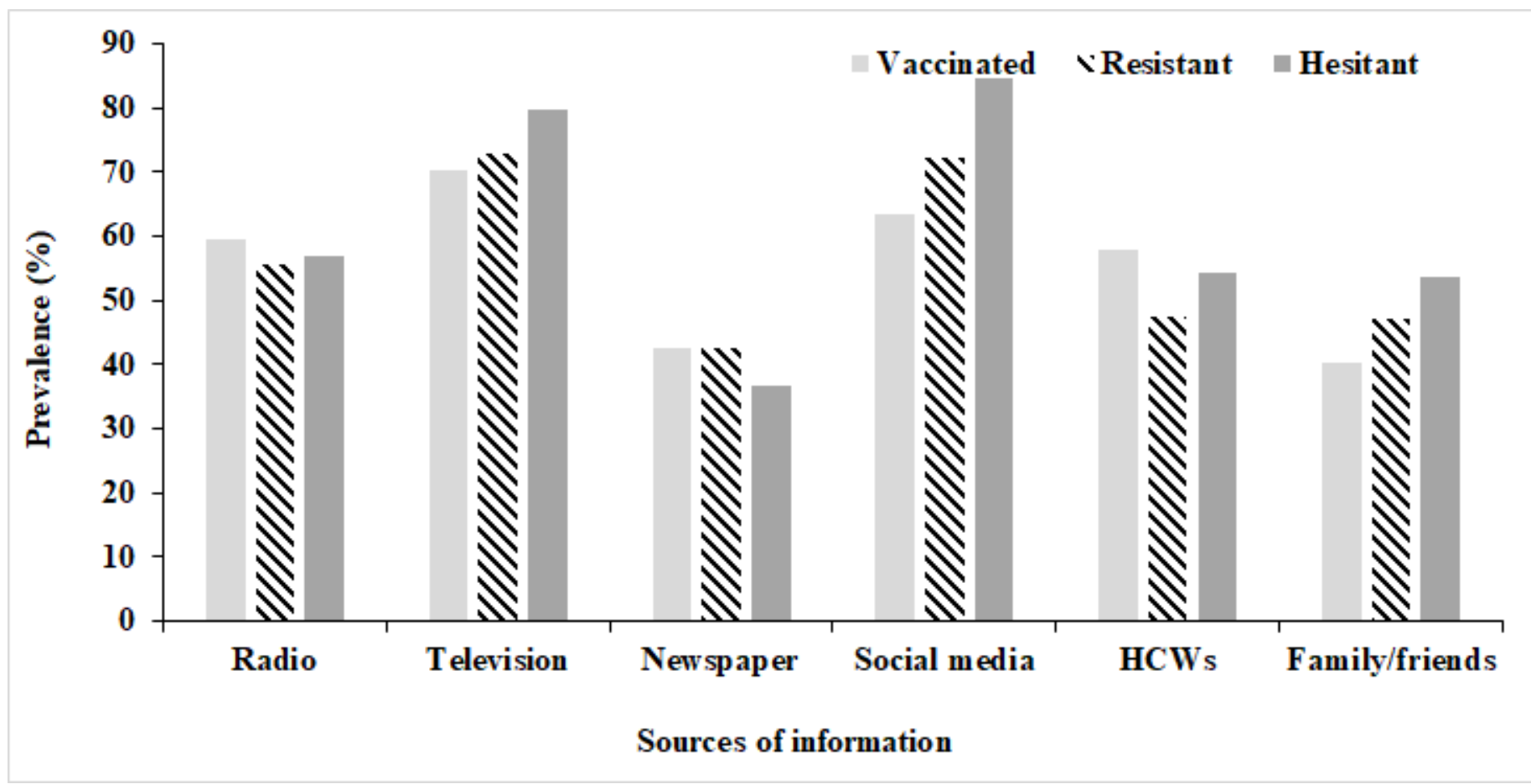

\section{Figure 1}

Prevalence of COVID-19 vaccination, hesitancy, resistance by information sources in sub-Saharan Africa during the lockdown. 


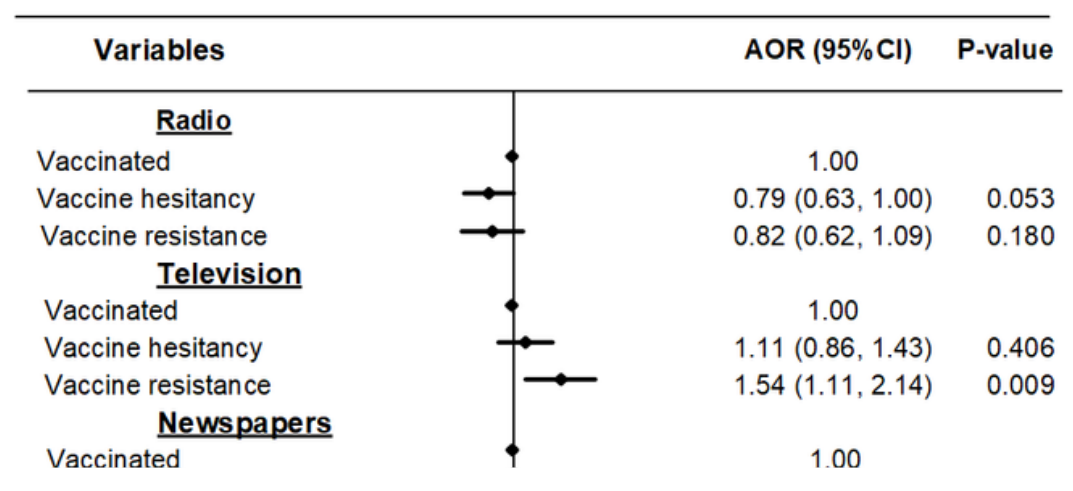

\section{Figure 2}

Association between information sources and vaccine hesitancy and resistance among SSAs during the lockdown.

\section{Supplementary Files}

This is a list of supplementary files associated with this preprint. Click to download.

- SupplementaryFile.docx 